\title{
Rüdingers «Atlas des peripherischen Nervensystems des menschlichen Körpers» mit Photographien von Joseph Albert
}

Zur Frühgeschichte der medizinischen Photographie

Von Hans-Konrad Schmutz

Eine Vielzahl technischer Erfindungen und die damit verknüpfte Industrialisierung haken das 19. Jahrhundert maßgeblich geprägt. Dies gilt auch für das Druckwesen. Mehrere Druckverfahren wurden in rascher Folge entwickelt. Einige davon benützten Anatomen nach auffallend kurzer Zeit für ihre Publikationen. So erschien das erste mit Lithographien illustrierte Handbuch der Humananatomie schon $1815^{1}$. Alois Senefelder hat sein «Lehrbuch der Steindruckerei» erst drei Jahre später geschrieben.

Die Photographie gehört zu den großen Erfindungen der ersten Hälfte des Jahrhunderts. Mit ihrer Einführung änderte sich die Sehweise für Generationen. Die Erfindung der Möglichkeit, ein durch Licht erzeugtes Bild zu fixieren, ist mit einer Reihe von Namen verknüpft. Nebst Arbeiten von Talbot und Niepce erlangte das Verfahren von Louis-Jacques-Mandé Daguerre (1787-1851) besondere Bedeutung. In der Sitzung vom 7.Januar 1839 berichtete der Physiker François Arago (1786-1853) erstmals davon in der französischen Akademie der Wissenschaften zu Paris. Bei Naturwissenschaftlern und Medizinern stießen diese frühen Daguerreotypien auf lebhaftes Interesse. So gehört beispielsweise der Berner Veterinäranatom Friedrich Gerber (1797-1872) zu den unabhängigen Miterfindern ${ }^{2}$. Begeistert schrieb auch Alexander von Humboldt an Carl Gustav Carus: «Es ist dies jedenfalls eine der freundlichsten und bewunderungswürdigsten Entdeckungen unserer Zeit... Arago hat jetzt das Geheimnis von Herrn Daguerre erhalten und hat in zehn Minuten ein vollendetes Bild unter seinen Augen entstehen sehen. Das Bild zeigt einen feinen Ableiter, den Arago mit bloßem Auge nicht gesehen hatte... Der geheimnisvolle chemische Überzug ist so lichtempfänglich, daß am Tage meiner Abreise Daguerre uns nach dem Observatorium das Bild der Mondscheibe brachte, ein Porträt, von Luna selbst hervorgebracht. ${ }^{3}$

Innerhalb der Anatomie wurden bald erste Erfolge in der mikroskopischen Anwendung erzielt ${ }^{4}$. An eine direkte Anwendung für die Buchillustration war noch nicht zu denken, da es sich bei den Daguerreotypien durchwegs um Unikate handelte. Es wurden zwar mehrere Versuche bekannt, die erzeugten Bilder zu 
ätzen und zu drucken, doch ergaben sich kaum brauchbare Lösungen. Selbst Alfred Donné (1801-1878), der sich sehr um diese Fragen bemüht hatte, konnte seinen Atlas nur mit der Nachhilfe des Stechers Oudet illustrieren ${ }^{5}$. Eine deutliche Verbesserung brachte die Photographie auf Papier. Über ein Positiv/NegativVerfahren war es William Fox Talbot (1800-1877) schon früh gelungen, identische Kopien von derselben Vorlage zu gewinnen. Anfänglich wurden Papiernegative verwendet. Später gab man durchsichtigen Negativträgern den Vorzug. Benützt wurden Glasplatten, die mit einem Überzug von jodhaltigem Eiweiß (nach Abel Niepce de Saint-Victor) oder mit einer jodhaltigen Collodionschicht (nach Frederick Scott Archer) versehen wurden ${ }^{6}$. Nun konnten Kopien in beliebig hoher Zahl hergestellt werden. Zudem hatte das Einzelbild eine weitaus bessere Qualität gewonnen.

Damit waren alle Voraussetzungen gegeben, Bücher mit Photographien zu illustrieren. So erschien 1844 in London H. Fox Talbots "The Pencil of Nature" und 1853 in Paris Renards Album «Le Paris photographié». ${ }^{7}$ Der erste Teil von Rüdingers Atlas erschien acht Jahre später. Es handelt sich also um eine sehr frühe, wenn nicht gar um die erste Topographisch-anatomische Anwendung. Das Tafelwerk mit insgesamt 46 eingeklebten großformatigen Aufnahmen erschien zwischen 1861 und 1867 unter dem Titel «Atlas des peripherischen Nervensystems des menschlichen Körpers ». ${ }^{8}$ Autor des Bandes war der Münchner Prosektor Nikolaus Rüdinger (1832-1896) ${ }^{9}$. Der bayrische Hofphotograph Joseph Albert (1825-1886) besorgte die photographische Wiedergabe der Präparate ${ }^{10}$.

\section{Das Werk}

Es war wesentlich für den späteren Erfolg des Werkes, daß kein Geringerer als Albert die Aufnahmen besorgte. Die hohe Qualität der Illustrationen wurde von manchem Rezensenten gelobt und überrascht noch heute.

Erste Abzüge hat Rüdinger bereits 1860 an die Wiener Akademie der Wissenschaften gesandt. Der Anatom Joseph Hyrtl besprach die großformatigen Positive in der Sitzung vom 18. Oktober 186011. Nach einer Notiz in der Wiener Medizinischen Wochenschrift dürfte es sich dabei um Probeabzüge für den geplanten Atlas gehandelt haben ${ }^{12}$. Auch deutet das große Format der gezeigten Bilder auf Albert als Photographen hin. Ein erster Teil erschien 1861. Das beigefügte Vorwort stammt aus der Feder des Münchner Ordinarius Theodor Ludwig Wilhelm Bischoff. Das verlegerische Risiko trug die literarisch-artistische Anstalt der J. G. Cotta'schen Buchhandlung in München. Das ganze Werk gliedert sich in 


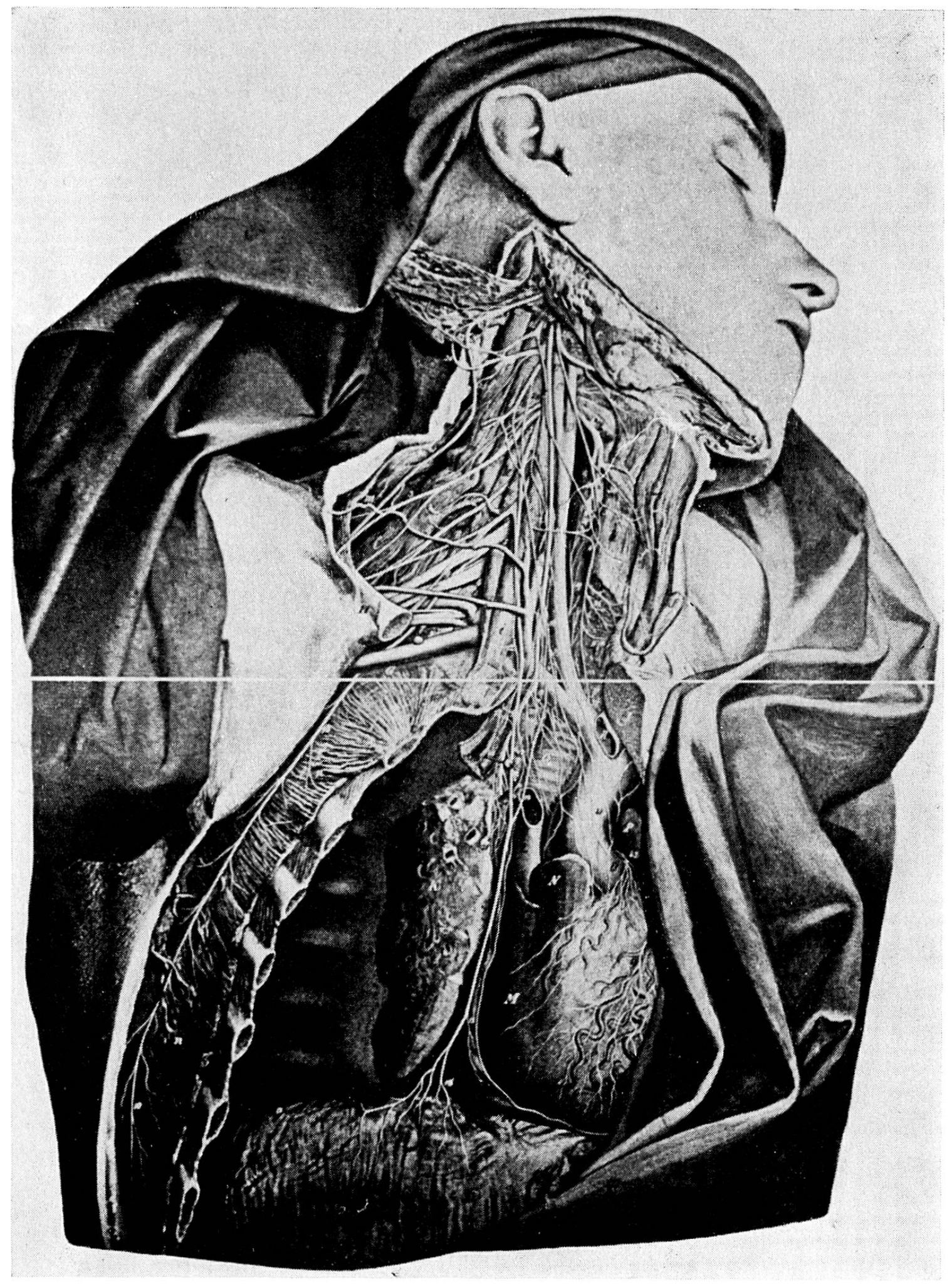

Abb.1. Fig. XXXXIII aus der neunten Lieferung, 1865; Titel: «Die Hals- und Herznerven.» Format: $52 \times 39 \mathrm{~cm}$ 
zehn Lieferungen, die, auf mehrere Mappen verteilt, in unregelmäßiger Folge erschienen.

Die ersten beiden Lieferungen zeigen die Austrittsstellen und den Verlauf der Hirnnerven. 1863 erschienen Abbildungen des Nervus hypoglossus und des Nervus olfactorius. Weiter wurden Präparate des eröffneten Wirbelkanals und der Spinalnerven berücksichtigt. Die fünfte und sechste Folge sind mehrheitlich der Beschreibung des Plexus cervicobrachialis und dessen Ästen gewidmet. Die beiden anschließenden Sendungen enthalten Darstellungen des Plexus lumbosacralis. Ein weiterer Abschnitt erreichte den Leser erst nach einer Zeitspanne von zwei Jahren. In dieser Mappe befindet sich eine besonders geglückte Aufnahme unter dem Titel «Die Hals- und Herznerven» (vgl. Abb.1). Nach einem erneuten Unterbruch wurde der Atlas 1867 abgeschlossen. Diese letzte Mappe enthält eine Serie von Bildern zur Erläuterung des peripheren vegetativen Systems.

Wie erwähnt, liegt der Wert des Atlas weniger im Text, der auf knappe Bildlegenden beschränkt ist, als vielmehr in den 46 meisterhaften Abbildungen begründet (kleinstes Format: 25,8 $\times 20,7 \mathrm{~cm}$; größtes Format: $30 \times 80 \mathrm{~cm}$ ). Alle sind auf Halbkarton aufgeklebt (Format: $36 \times 51 \mathrm{~cm}$ ). Aus elf Photographien, die beträchtlich größer sind als die Mappen, wurden Falttafeln angefertigt (vgl. Abb.1). Obwohl viele Aufnahmen stark retuschiert sind, lassen sie sich qualitativ durchaus mit wesentlich später veröffentlichten Photographien vergleichen.

Stilistisch können die Bilder in zwei Gruppen unterteilt werden. Die meisten Aufnahmen sind sehr nüchtern gehalten. In ihnen sucht man vergebens nach einer idealisierenden Pose. Die Forderung nach einer möglichst naturgetreuen Wiedergabe findet sich auch im Text. Bischoff schrieb in der Einleitung: «So ist wie gesagt in diesen Darstellungen eine Naturtreue und Wahrheit erreicht worden, deren sich keine bisher jemals gelieferten anatomischen Abbildungen erfreuten... Ich halte dafür, daß vollkommenste Naturtreue, Wahrheit, Klarheit und Deutlichkeit der einzige Zweck anatomischer Abbildungen sein kann.» ${ }^{13}$ Diese Auffassung widerspiegelt sich in der Mehrzahl der Aufnahmen. Zum Beispiel sei auf die Tafel XXXXV der zehnten Lieferung verwiesen (Abb.2). Auf schmückendes Beiwerk wurde verzichtet. Abdeckende Tücher oder Instrumente wurden entweder nicht mitphotographiert oder fielen dem Retuschierpinsel zum Opfer.

Dagegen zeigen einige wenige Aufnahmen Stilelemente, die an Kupferstiche aus Atlanten des 18. Jahrhunderts erinnern. Dies läßt sich z.B. an der Tafel XXXXIII aus der neunten Lieferung nachweisen (vgl. Abb. 1) ${ }^{14}$. Diese Aufnahme gleicht einem Kupferstich, der von Christian Jeremias Rollin für Hallers Icones anatomicae gezeichnet worden war ${ }^{15}$. Beide Darstellungen zeigen die gleiche 


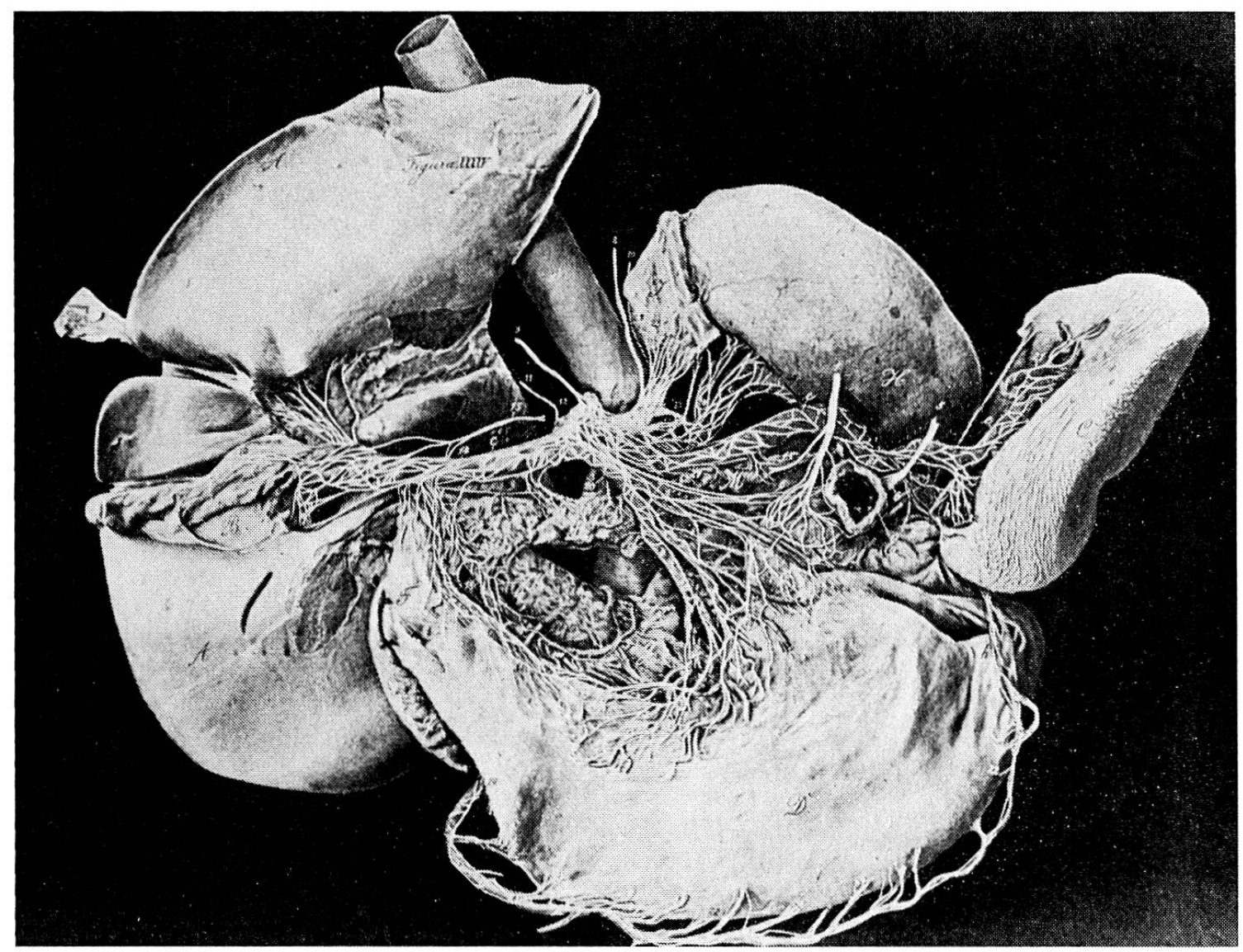

Abb.2. Fig. XXXXV aus der zehnten Lieferung, 1867; Titel: «Leber, Milz, Pankreas, Niere, Nebenniere und Magen im Zusammenhang mit ihren Gefäßen und Nerven unter Wasser und Weingeist dargestellt. » Format: $39 \times 29,5 \mathrm{~cm}$

Aufsicht auf den eröffneten Thorax. Störendes ist in beiden Fällen mit Tüchern abgedeckt. Allerdings verzichtet Albert auf einen stilisierten Hintergrund.

Erfolg des Werkes

Wissenschaftlich war der Atlas ein Erfolg. Wie schon vermerkt wurde, hatte sich Joseph Hyrtl 1860 hinter die Bemühungen der beiden Münchner gestellt. Jacob Henle schrieb nach dem Erscheinen der ersten Lieferung: «Übertrifft an Naturtreue und Anschaulichkeit Alles, was Kupferstich und Lithographie bisher in diesem Gebiet geleistet haben. » ${ }^{16}$ Für die Zeitschrift «Lancet » hielt ein Rezensent fest: «... the plates are perfectly wonderful as to exactitude and finish ». ${ }^{17}$ Später bemühte sich besonders Joseph Albert, den Atlas bekannt zu machen. 1862 
sandte er Abzüge aus den ersten vier Lieferungen an die Industrieausstellung nach London ${ }^{18}$. Fünf Jahre später gab Rüdinger Präparate an die Weltausstellung in Paris ${ }^{19}$. 1868 fand in Hamburg die dritte deutsche photographische Ausstellung statt. Hier wurde Rüdinger in Anerkennung seiner Verdienste um die wissenschaftliche Anwendung der Photographie mit einem ersten Preis ausgezeichnet ${ }^{20}$.

$\mathrm{Zu}$ dieser Zeit waren zwei Mappen eines weiteren Werkes erschienen. (Rüdinger, Nikolaus, Atlas des menschlichen Gehörorganes, München 1866-1875). Dieser Titel fehlt im Werkverzeichnis des Hofphotographen ${ }^{21}$.

Trotz der Anstrengungen von Autor und Illustrator blieb der finanzielle Erfolg bescheiden. Die gewählte Abbildungsart war äußerst kostspielig. Daraus ergab sich mit 64 bayrischen Gulden ein ungewöhnlich hoher Verkaufspreis ${ }^{22}$. Für die Mehrzahl der praktizierenden Ärzte war das Werk unerschwinglich ${ }^{23}$. Deshalb ging Rüdinger daran, billigere Zweitfassungen herauszugeben. Ein erster Titel erschien noch im Jahr der Hamburger Ausstellung ${ }^{24}$. Der Text blieb wiederum auf knappe Bildlegenden beschränkt. An die Stelle der aufwendigen Photographien traten Stahlstiche. Zehn der insgesamt zwölf Tafeln signierte Arno Meermann (1829-1908) ${ }^{25} .1870$ erschien ein weiterer Band mit der Überschrift «Die Anatomie der menschlichen Rückenmarks-Nerven für Studierende und Ärzte ». ${ }^{26}$ Meermann hatte für diese Publikation 27 Abzüge aus den Lieferungen drei bis zehn bearbeitet. Allerdings wirken die Stahlstiche etwas verwaschen. Der Stecher vermochte die Qualität der Albertschen Aufnahmen nur teilweise wiederzugeben. Noch für zwei weitere Publikationen hat Rüdinger auf Abbildungen aus dem «Atlas des peripherischen Nervensystems» zurückgegriffen. Sechs Photographien fügte er der dritten Lieferung des «Atlas des menschlichen Gehörorganes» bei, und eine der Abbildungen in seinem 1891 gedruckten «Cursus der topographischen Anatomie» läßt sich auf ein bereits 1863 veröffentlichtes Bild des weiblichen Beckens zurückführen ${ }^{27}$.

Daß Rüdinger darauf verzichtete, seinen späteren Werken Papierabzüge beizufügen, entspricht der allgemeinen Entwicklung. Nicht zuletzt aus finanziellen Überlegungen gab man dem Stahl- und Holzstich den Vorzug. Zudem wurden mehrere Lichtdruckverfahren bekannt. Joseph Albert hat sich mit seiner 1868 veröffentlichten «Albertotypie» selbst tätig an dieser Entwicklung beteiligt.

Rüdingers Leistungen wurden in späteren Jahren hauptsächlich in photographischen Lehrbüchern gewürdigt. Siegmund Stein bezeichnete in seinem 1877 erschienenen Handbuch den Atlas als beispielhaft ${ }^{28}$. Noch 1911 fügte Gustav Fritsch einem Aufsatz die Reproduktion eines Abzuges aus dem damals bereits 50 Jahre alten Werke bei ${ }^{29}$. 


\section{Anmerkungen}

1 Münz, M., Handbuch der Anatomie des menschlichen Körpers mit Abbildungen/Muskellehre mit Abbildungen nach Albin, Landshut 1815.

Herrlinger, R., Das erste lithographisch illustrierte Lehrbuch der Anatomie. Sudhoffs Archiv 47 (1963), S.224-236.

${ }^{2}$ Baier, W., Geschichte der Fotographie, München 1975, S. 92 (zit. Baier).

${ }^{3}$ Aus einem Brief vom 25. Februar 1839; vgl. Baier, S.116-117.

${ }^{4}$ Gernsheim, A., Medical Photography in the nineteenth century. Med. Biol. Illustr.11 (1961), S. 85-92, 147-156.

5 Donné, A., et Foucault, L., Cours de Microscopie complémentaire des études médicales, Anatomie microscopique et physiologie des fluides de l'économie, Atlas exécuté d'après nature au microscope-daguerréotype, Paris 1845.

Kraus, R.H., Photographs as Early Scientific Book Illustrations. History of Photography 2 (1978), S. 292.

${ }^{6}$ Gerlach, J., Die Photographie als Hülfsmittel mikroskopischer Forschung, Leipzig 1863, S. 2-3.

Burns, S. B., Early Medical Photography in America (1839-1883), I. History of photographic processes. N. Y. State J. Med. 79 (1979), S. 788-795; hier S. 790.

7 Schultze, R.S., Books illustrated with Original Photographs: Notes on a Collection and Bibliography, in: Helwich, O., Jubiläums-Festschrift, Hundert Jahre Photographische Gesellschaft in Wien, 1861-1961, Wien 1961, S. 145, und Dumaître, P., Histoire de la médecine et du livre médical, Paris 1979, S.365-366.

${ }^{8}$ Rüdinger, N., Atlas des peripherischen Nervensystems des menschlichen Körpers, mit einem Vorwort von Professor Dr. Th. W.L. Bischoff, nach der Natur photographirt von Joseph Albert, K. B. Hofphotograph in München, München 1861-1867.

1.Lieferung $1861 \quad$ 6. Lieferung 1863

2.Lieferung $1861 \quad$ 7.Lieferung 1863

3. Lieferung $1862 \quad$ 8. Lieferung 1863

4. Lieferung $1862 \quad$ 9. Lieferung 1865

5. Lieferung $1863 \quad$ 10. Lieferung 1867

${ }^{9}$ Kupffer, C. von, Nikolaus Rüdinger. Anat. Anz.13 (1897), S.219-232.

10 Ranke, W., Joseph Albert - Hofphotograph der bayerischen Könige, München 1977.

${ }^{11}$ Sitzungsberichte der Mathematisch-Naturwissenschaftlichen Classe der Kaiserlichen Akademie der Wissenschaften, Bd.42, Wien 1861, S.221.

12 Wien med. Wschr.11 (1861) S.547.

${ }^{13}$ vgl. Bischoff, Th.W.L., im Abschnitt Vorwort/Préface zur ersten Lieferung von 1861.

${ }^{14}$ Zur Buchillustration im 18. Jahrhundert vgl. Mann, G., Medizinisch-naturwissenschaftliche Buchillustration im 18. Jahrhundert in Deutschland. Marb. Sitzungsber.86 (1964), S. $3-48$.

${ }^{15}$ vgl. Haller, A., Icones anatomicae, 8 Lieferungen, Göttingen 1743-1756, Fasc. II, Tab. II, und Schmutz, H.-K., Barocke und klassizistische Elemente in der anatomischen Abbildung. Gesnerus 35 (1978), S.54-65, Abb.3.

${ }^{16}$ Henle, J., Berichte über die Fortschritte der Anatomie und Physiologie im Jahre 1860, Leipzig und Heidelberg 1862, S.135. 
17 The Lancet (1861), S.310.

${ }^{18} \mathrm{vgl}$. Arztliches Intelligenz-Blatt 9 (1862), S.231, und Official Catalogue of the International Exhibition, London 1862, S.254.

${ }^{19}$ vgl. Arztliches Intelligenz-Blatt 14 (1867), S.306-307, und Notiz in Le Temps vom 12. Mai 1867.

${ }^{20}$ Photographische Correspondenz 5 (1868), S.277. - Joseph Albert hatte als Obmann der photographischen Jury-Commission die ausgestellten Arbeiten mit zu begutachten!

${ }^{21}$ Aus der Zusammenarbeit des Photographen mit Rüdinger vermerkt Ranke lediglich den «Atlas des peripherischen Nervensystems des menschlichen Körpers».

${ }^{22}$ Zum Wert des bayrischen Guldens vgl.: Winkler, R.A., Die Frühzeit der deutschen Lithographie, München 1975, S.436.

${ }^{23}$ vgl. Ärztliches Intelligenz-Blatt 15 (1868), S.184.

${ }^{24}$ Rüdinger, N., Die Anatomie der menschlichen Gehirn-Nerven, München 1868.

${ }^{25}$ Thieme, U., und Becker, F., Allgemeines Lexikon der bildenden Künstler, Bd. 24, Leipzig 1942, S. 333.

${ }^{26}$ Rüdinger, N., Die Anatomie der menschlichen Rückenmarks-Nerven, Stuttgart 1870. (Dieser Band war mit einem Verkaufspreis von $9 \mathrm{fl}$. bedeutend billiger als das Hauptwerk.)

${ }^{27}$ Als Vorlage für die Abb.56 muß die Fig. XXXII aus der Doppellieferung fünf und sechs von 1863 gedient haben.

${ }^{28}$ Stein, S.Th., Das Licht im Dienste wissenschaftlicher Forschung, Leipzig 1877, S. 307.

${ }^{29} \mathrm{vgl}$. Fritsch, G., in: Wolf-Czapek, K. W., Angewandte Photographie in Wissenschaft und Technik, Berlin 1911, S.II, 67-72, Tafel 26, Fig. 78. (Diese Abbildung entspricht der Fig. XII aus der zweiten Lieferung von 1861.)

\section{Verdankung}

Herrn Prof. Huldrych M.Koelbing möchte ich für die freundlich gewährte Unterstützung sowie für die Durchsicht des Manuskriptes herzlich danken.

\section{Summary}

The «Atlas des peripherischen Nervensystems des menschlichen Körpers» has been published between 1861 and 1867. It is one of the earliest anatomical books that has been illustrated with original photographs. The anatomist Nikolaus Rüdinger of Munich is the author. The 46 pictures were taken by the photographer of the Bavarian Court, Joseph Albert. The illustrations are of high quality and can be compared with those of other works, published much later.

Hans-Konrad Schmutz

Abteilung für Makroskopie des Anatomischen Institutes (Leitung: Prof. Dr. med. St. Kubik)

Gloriastraße 19

CH-8006 Zürich 